\title{
GALECTIN-3: MULTIPLE CLINICAL APPLICATIONS
}

\author{
Antoniya Kisheva* and Yoto Yotov \\ First Department of Internal Diseases, ES Cardiology, Medical University Varna, Bulgaria
}

Galectin-3 is a $\beta$-galactoside binding lectin, containing carbohydrate-recognition domain, which interacts with a number of ligands. It is found in many tissues and is distributed intra-and extracellularly. The localization of the biomarker determines its function. Galectin-3 is involved in variety of biological processes sush as inflammation, fibrosis, immunological response and neoplastic growth. It may be used as a diagnostic or prognostic biomarker especially in cancer and cardiovascular diseases. The present review summarizes some of the properties of this biomarker. Biomed Rev 2019;30:83-88

Keywords: Galectin-3, inflammation, fibrosis, cancer, cardiovascular diseases, microtubules

\section{INTRODUCTION}

Galectins are a multifunctional family of soluble $\beta$-galactoside binding lectines, which play an important role in inflammation, fibrosis, immunological response and neoplastic growth. They use specific carbohydrate-recognition domain (CRD) to bind carbohydrate molecules (1). At present, 15 galectins have been identified, which are classified into three subgroups, according to protein structure and domain organization $(1,2)$. The prototypical galectins, galectin-1, $-2,-5$, $-7,-10,-11,-13,-14$ and -15 , contain single CRD. The tandem-repeat galectins, galectin- $4,-6,-8,-9$ and -12 , are with two CRD separated by a linker region in a single polypeptide chain. Galectin-3 (Gal-3) is the only chimera-type ga- lectin, which contains CRD connected to flexible non-lectin 110-130 amino acids N-terminal domain, consisting of 7-14 tandem short amino acid segments. It is a $29-35 \mathrm{kD}$ protein. It interacts with a number of ligands - carbohydrates like $\mathrm{N}$-acetylgalactosamin, and non glycosylated molecules like surface cell receptors (macrophages) and extracellular receptors (collagen type IV). C-terminal domain is responsible for the $\beta$-galactoside binding and anti-apoptotic activity and the presence of $\mathrm{N}$-terminal domain is necessary for the secretion, translocation, multimerization of Gal-3 and also for the antiapoptotic activity (1-3).

Galectin-3 is the best studied member of galectins family. In humans Gal-3 is distributed in many tissues, preferably

Received 10 December 2019, revised 19 December 2019, accepted 20 December 2019.

*Correspondence to: Dr Antoniya Kisheva, 23 Knyaz Nikolaevich Str., Entr 2, BG-9002 Varna, Bulgaria.

Tel.: +359 887425 210; E-mail: tony_kisheva@yahoo.com 
epithelia - skin, mucosa, epithelial lining of the digestive and respiratory tract, urothelium and excretory tubes of the kidney, in the myocardial cells, liver, in the fibroblasts, chondrocytes, osteoblasts, osteoclasts, keratinocytes and Schwann cells. Gal-3 is involved in immune response. It is expressed in neutrophils, eosinophils, basophils, mast cells, Langerhans cells, dendritic cells, monocytes and macrophages. The biomarker is found in a number of tumors and the expression reflects the tumor characteristics. Galectin- 3 can be located intracellularly in the cytoplasm, nucleus and membrane and extracellularly. It is secreted in the interstitium and in the blood flow from activated macrophages in response to physiological or pathophysiological stimuli . The different distribution of Gal-3 is associated with its various functions.

\section{ROLE OF GALECTIN-3 IN INFLAMMATION}

Galectin-3 participates in acute and chronic inflammation. Recent studies have demonstrated that Gal-3 can detect certain microbial structures by binding specific carbohydrate structures of glycoproteins and glycolipids from many pathogens $(3,4)$. It acts pro-inflammatory stimulating the infiltration of neutrophils and other immune cells to the infected sites and inducing production of inflammatory cytokines from the macrophages. The biomarker can also be released in response to damage. Galectin-3 is synthesized and accumulated in the cytoplasm of cells, separately from the glycan ligands. Different pathogens may trigger its secretion. It may be passively released from damages cells or actively secreted from activated cells by receptor engagement of cytokines (6). Galectin-3 is secreted through a leaderless pathway, which requires specific amino-acid sequence close to the Nterminal portion of the protein. The release of Gal-3 from the damaged cells correlate with the level of virulence of the microorganism. There are data in mice that Gal-3 is exported to the alveoli when the microbic agent is pathogenic like S. pneumonia, but not in infection with $E$. coli, which is less pathogenic $(6,7)$. Galectin-3 can act as pattern-recognition receptor (PRR) in infectious diseases, caused by bacteria, viruses, fungi and parasites ${ }^{10}$. It interacts with microbial ligands by binding lipopolysaccharides (LPS). Studies on infection with Escherichia coli and Pseudomonas aeruginosa reveal two independent binding sites on Gal-3 (8). One is the carbohydrate binding site in the $\mathrm{C}$-terminal domain, which interacts with beta-galactoside-containing polysaccharide chain. The other site is located on the N-terminal domain and binds to the inner core of LPS. Both interactions lead to oligomerization of Gal-3 and induce the proinflammatory activity on the neutrophils. Macrophages from Gal-3-deficient mice produce more cytokines in response to LPS stimulation. The process is suppressed by the addition of exogenous Gal-3, showing that galectin-3 is a natural negative modulator for LPS function (7). Galectin-3 also recognizes and binds the mycolic acid from mycobacteria (7). This results in inhibition of oligomerization of Gal-3 but does not affect the carbohydrate-binding abilities. Another mechanism of interaction is the accumulation of phagosomes, containing live mycobacteria, primarily at the cytosolic face of the phagosome membrane (7). The recognition of pathogenic fungi from Gal-3 is mediated by Dectin-1. It is a macrophage transmembrane lectin on the fungal cell wall, which is a receptor for b-glucans. Gal-3 binds to oligomannas on the cell wall. Macrophages must express both Dectin-1 and Gal-3 as in Candida albicans, to release a response. They secret tumor necrosis factor- $\alpha(\mathrm{TNF}-\alpha)$ in a C.albicans-specific manner through cross-talk with Toll-like receptor 2 (TLR2) . Additionally binding of Gal-3 to $\beta-1,2$ oligomannas of $C$. albicans kills fungi directly . Gal-3 has antimicrobial activity only to species, containing $\beta-1,2$ oligomannas. Gal-3 interacts with parasites like Leishmania $(9,10)$ and Trypanosoma (9) cruzi . The immunological response to infection with Leishmania major is performed through specific depletion of Gal-3. Binding of Gal-3 to the polygalactosyl repeats of Leishmania leads to truncation of Gal-3 by their surface major protease, gp63. The truncated Gal-3 cannot oligomerize or form stable Gal-3 association (9). Galectin-3 interacts with Trypanosoma cruzi by binding three specific proteins of the parasite. The binding of $T$. cruzi to human coronary artery smooth muscle cells is also Gal-3 dependent (10).

Galectin-3 plays a role in inflammation also in noninfectious conditions. Recently the metabolic inflammation has raised the scientific interest. It can occur as a result of adipose tissue expansion in obesity and precedes the development of insulin resistance, type 2 diabetes and nonalcoholic fatty liver disease . Galectin-3 participates in the pathogenesis of diabetic complications via its ability to bind advanced glycation end-products (AGEs) and advanced lipoxidation end-products (ALEs). Yilmaz et al found that Gal-3 levels were significantly higher in patients with diabetes than those without and higher in prediabetic than nondiabetic group. In the same study Gal-3 correlated with fasting plasma glucose $(r=0.787, P<0.01), 2$-hour plasma glucose $(r=0.833, P<$ $0.01), \mathrm{CRP}(r=0.501, P<0.01)$ and insulin resistance (HO- 
MA-IR) index $(r=0.518, P<0.01)$. A small study showed higher levels of Gal-3 in patients with gout, but further research is needed (11).

\section{ROLE OF GALECTIN-3 IN CANCER}

Galectin-3 is implicated in carcinogenesis and tumor progression through different mechanisms. In cancer, it has both intracellular and extracellular functions. The glycoprotein participates in cell proliferation, adhesion, differentiation, angiogenesis and apoptosis in normal and pathologic tissues. It impacts the tumor cell transformation, migration, invasion and metastasis (12).

Overexpression of Gal-3 is found in many cancers and it has the potential to be used as an oncological biomarker for diagnosis. There is evidence that Gal-3 over expression is associated with neoplastic transformation $(11,12)$. Suppression of Gal-3 in breast carcinoma cells causes a reversion of the transformed phenotype as determined by altered morphology, loss of serum-independent growth, acquisition of growth inhibition properties by cell contact, and abrogation of anchorage-independent growth . Thyroid papillary carcinoma cells lose their characteristic transformed phenotypes in cell culture after inhibition of Gal-3 expression . The transfection of Gal-3 cDNA into normal thyroid follicular cells induced serum-independent growth, clonogenicity in soft agar and loss of contact inhibition. The resistance of tumor cells to apoptosis plays an important role for the tumor malignancy and progression. It is shown that Gal-3 has an anti-apoptotic and/or pro-apoptotic features in different cell types and localizations. Several regulatory pathways for the antiapoptotic activity are discussed. In response to apoptotic stimuli galectin-3 translocates from the cytosol or the nucleus to the perinuclear mitochondrial membrane, prevents mitochondrial damage and suppresses cytochrome c release. This process leads to down-regulation of caspase activation. The phosphorylation of Gal-3 at position $\operatorname{Ser}^{6}$ also contributes for the anti-apoptotic activity. Other possible mechanisms are the activation of ERK and JNK (c-Jun NH2-terminal kinase) pathways and interaction with CD95 (APO-1/Fas) receptor . Extracellular Gal-3 is found to have pro-apoptotic activity in human leukemia cell lines and human peripheral blood mononuclear cells . Galectin-3 participates in the immune regulation and thus influences immunosuppression in tumorogenesis. It acts by suppressing CD8+ cells via LAG-3 and inhibiting expansion of plasmocytoid dendritic cells in patients with pancreatic ductal adenocarcinoma. In combination with soluble fibrinogen Gal-3 is found to modulate the functionality and life-span of the neutrophils . Galectin-3 plays a role in tumor metastasis. When located subcellularly it regulates specific gene expression by transcription factors such as $\beta$-catenin (21).. Some integrins ( $\alpha 1 \beta 1, \alpha 4 \beta 7, \alpha 6 \beta 1$, $\alpha \mathrm{M} \beta 1$, etc.) can also be receptors for Gal-3 (22).. It increases heterotypic tumor cell adhesion to endothelial cells by binding to cancer associated TF/MUC1 and mediates homotypic aggregation of tumor cells forming tumor micro-emboli and invading blood vessels to reach distant organ sites $(23,24)$. Angiogenesis is essential for tumor invasion and metastasis. It is suggested that Gal-3 modulates vascular endothelial growth factor- and basic fibroblast growth factor-mediated angiogenesis (25). Another study showed regulation of angiogenic and migratory response in endothelial cells via modulation of integrin-linked kinase signaling (26).. An interaction of Gal-3 with endothelial cell surface enzyme aminopeptidase N/CD13 has also been reported to influence endothelial vascularization in the early steps of angiogenesis (27).. Galectin-3 overexpression in the tumor caused macrophage infiltration and acceleration of angiogenesis (28).. Because Gal-3 reflects dynamic changes in cancer status, it may be used not only as marker of diagnosis, but also as a marker of cancer progression and prognosis 129 ).

\section{ROLE OF GALECTIN-3 IN CARDIOVASCULAR DISEASES}

Galectin-3 as a marker of fibrosis and inflammation plays an important role in cardiovascular diseases. It is found at the site of fibrosis together with fibroblasts and macrophages ${ }^{29}$. In vitro experiments on cultures of cardiac fibroblasts show that Gal-3 induces proliferation and synthesis of collagen . It is a potent mitogenic factor for fibroblasts, affects synthesis of new matrix components as collagen type 1 and influences the degradation of components of extracellular matrix by metalloproteinases.

Galectin-3 has not only prognostic significance, but is also a target for acute and chronic intervention. The participation of Gal-3 in the progression of heart failure (HF) is first studied in animals by Sharma et al (12). Higher levels of the protein were found in patients with severe cardiac fibrosis. To determine whether Gal-3 is directly associated with fibrosis, it was injected intrapericardial in normal murine hearts. This led to reduction of left ventricle ejection fraction (LV EF), higher LV mass and abundance of collagen type I in comparison with placebo. These results are confirmed with human myocardial biopsy, where higher levels of Gal-3 correlated with lower LV EF.

Galectin-3 is studied in a number of cardiovascular clini- 
cal trials. It predicts the all cause mortality in the general population and the levels correlate positively with age and cardiovascular risk factors as diabetes, smoking, angina, hypertension, obesity and hyperlipidemia. This is more pronounced in females in PREVEND (Prevention of Renal and Vascular End-stage Disease) study . Results of PROVE-IT TIMI (Pravastatin or Atorvastatin Evaluation and Infection Therapy - Thrombolysis in Myocardial Infarction) show that Gal-3 is associated with higher risk of developing HF after acute coronary syndrome (33).. In DEAL-HF (DeventerAlkmaar heart failure) Gal-3 was an independent predictor for mortality in patients with moderate and severe HF. The levels of Gal-3 were associated with age, renal function and HF severity (34).. Sub analysis of $\mathrm{COACH}$ (Coordinating Study on Outcomes of Advising and Counselling in Heart Failure) trial demonstrated that Gal-3 is an independent prognostic marker for $\mathrm{HF}$ and may be especially useful in HF with reduced EF (32). . In the same study patients with higher Gal-3 levels were more likely to suffer from diabetes or atrial fibrillation $(\mathrm{AF})$.

Routine measurement of Gal-3 in HF patients has a number of benefits 4 . Heart failure may be divided into two groups according to involvement of Gal-3 in the pathogenesis. Galectin-3 mediated HF is characterized with high activity of fibrogenesis, progressive course and high risk of adverse outcome. The other group is with heterogenic etiology and pathology, slow progression and low risk of adverse outcome (35). Gal-3 is complementary to brain natriuretic peptides (BNP), but is independent predictor. Patients with increase of both biomarkers have the worst outcome (36).. Most of the biomarkers as BNP and CRP are released in the circulation as a result of cell damage. In contrast, Gal-3 is found in the extracellular matrix and plays a causative role in the remodeling process. Its inhibition may stop or regress the fibrosis. Galectin-3 testing by enzyme-linked immune-sorbent assay (ELISA) is approved by the US Food and Drug Administration (FDA) in chronic heart failure patients and is included in the guidelines (37).

The hallmark of AF is structural remodeling due to pro-inflammatory and profibrotic changes in the atrial tissue, which supposes an association between Gal-3 and AF. Data from Framingham study derived from 3000 patients show that higher circulating levels of Gal-3 may predict AF (37).. The prevalence of AF was higher according to age specific quartiles $-3,7 \%, 5,9 \%, 9,1 \%$ and $11,5 \%$ respectively in 10 years, but after adjusting for traditional risk factor the significance is lost. Similar are the results of Somnez et al (38). They found higher levels of Gal-3, matrix metalloproteinases and type III procollagen peptide in AF patients. Studies about the role of Gal-3 as a prognostic marker for AF recurrences after catheter ablation are controversial. Wu et al measured higher Gal-3 levels in patients with persistent AF without structural heart disease and demonstrated that the biomarker significantly predicts AF recurrence after ablation (39).. Kornej et al also found higher values of Gal-3 in AF patients, but thought this is attributable to the accompanying cardiometabolic diseases, but not to the arrhythmia itself. They conclude that Gal-3 cannot predict AF recurrence after ablation (40).. Galectin-3 could be the new target for upstream therapy in $\mathrm{AF}$, because it is a mediator of atrial fibrosis, which induces an anisotropic conductance in the atria (41).. A couple of studies show correlation between Gal-3 and thromboembolic risk as assessed by $\mathrm{CA}_{2} \mathrm{DS}_{2}$ Vasc score (42-44). .

\section{CONCLUSION}

Galectin-3 is expressed intra- and extracellularly in various tissues. It is involved in multiple biological processes, such as inflammation, oncogenesis, tumor progression, metastasis and angiogenesis. Because of its pro-inflammatory and profibrotic activity, Gal-3 is used as a marker of prognosis in oncological and cardiovascular diseases, especially in $\mathrm{HF}$ and AF. More studies are needed to found the utility of the biomarker as a therapeutic target (see 45-47 for galectin and microtubules; cf 48-50).

\section{CONFLICT OF INTEREST}

The authors declare no conflict of interest.

\section{REFERENCES}

1. Dumic J, Dabelic S, Flögel M. Galectin-3: an openended story. Biochim Biophys Acta 2006; 1760:616-635. doi:10.1016/j.bbagen.2005.12.020

2. Fritsch K., Mernberger M., Nist A., Stiewe T., Brehm A, Jacob R. Galectin-3 interacts with components of the nuclear ribonucleoprotein complex, BMC Cancer, 2016; 16: 502. doi: 10.1186/s12885-016-2546-0.

3. Mishra BB, Li Q, Steichen AL, Binstock BJ, Metzger DW, et al. Galectin-3 functions as an alarmin: pathogenic role for sepsis development in murine respiratory tularemia. PLoS One, 2013; 8(3): e59616. doi:10.1371/journal. pone. 0059616

4. Vasta GR. Galectins as pattern recognition receptors: structure, function, and evolution. Adv Exp Med Biol 2012; 946:21-36. doi: 10.1007/978-1-4614-0106-3_2. 
5. Vasta GR. Roles of galectins in infection, Nat Rev Microbiol 2009; 7(6): 424-438. doi:10.1038/nrmicro2146.

6. Mey A., Leffler H., Hmama Z., Normier G, Revillard JP. The animal lectin galectin-3 interacts with bacterial mediators of inflammation lipopolysaccharides via two independent sites. J Immunol 1996; 156(4):1572-1577.

7. Fradin C, Jouault T, Mallet A, et al. Beta-1,2-linked oligoman-nosides inhibit Candida albicans binding to murine macrophage. J Leukoc Biol 1996; 60:81-87. https://doi. org/10.1002/jlb.60.1.81

8. Kohatsu L, Hsu DK, Jegalian AG, Liu FT, Baum LG. Galectin-3 induces death of Candida species expressing specific beta-1,2-linked mannans. J Immunol 2006; 177:4718-4726. doi:10.4049/jimmunol.177.7.4718

9. Radosavljevic GD, Pantić JM, Jovanović I, Lukic M, Arsenijević N. The two faces of Galectin-3: Roles in various pathological conditions. Ser J Exp Clin Res 2016; 17:187 - 198. doi: https://doi.org/10.1515/sjecr-2016-0011

10. Yilmaz H, Cakmak M, Inan O, Darcin T, Akcay A. Increased levels of galectin-3 were associated with prediabetes and diabetes: new risk factor? $J$ Endocrinol Invest. 2015; 38(5):527-33. doi: 10.1007/s40618-014-0222-2.

11. Honjo Y, Nangia-Makker P, Inohara H, Raz A. Downregulation ofgalectin-3 suppresses tumorigenicity of human breast carcinomacells, Clin Cancer Res. 2001; 7:661-668.

12. Yoshii $\mathrm{T}$, Inohara $\mathrm{H}$, Takenaka $\mathrm{Y}$, Honjo $\mathrm{Y}$, Akahani $\mathrm{S}$, et al. Galectin-3 maintains the transformed phenotypeof thyroid papillary carcinoma cells. Int J Oncol 2001; 18:787-792. doi:10.1016/s0304-3835(03)00056-9.

13. Takenaka Y., Inohara H., Yoshii T, et al. Malignant transformation of thyroid follicular cells by galectin-3, Cancer Lett. 2003; 195:111-119

14. Nakahara S, Oka N, Raz A. On the role of galectin-3 in cancer apoptosis, Apoptosis, 2005; 10:267-275 doi:10.1007/s10495-005-0801-y.

15. Yu F, Finley Jr RL, Raz A, Kim HR. Galectin-3 translocates to the perinuclear membranes and inhibits cytochrome $\mathrm{c}$ release from the mitochondria. A role for synexin in galectin-3 translocation J Biol Chem 2002; 277:15819-15827

16. Takenaka Y, Fukumori T, Yoshii T, et al. Nuclear export of phosphorylated galectin-3 regulates its antiapoptotic activity in response to chemotherapeutic drugs. Mol Cell Biol, 2004; 24: 4395-4406. doi:10.1128/mcb.24.10.43954406.2004

17. Fukumori T, Takenaka Y, Oka N, et al. Endogenous galectin-3 determines the routing of CD95 apoptotic signaling pathways. Cancer Res 2004; 64:3376-3379.
doi:10.1158/0008-5472.CAN-04-0336

18. Fukumori T, Takenaka Y, Yoshii T, et al. CD29 and CD7 mediate galectin-3-induced type II T-cell apoptosis. Cancer Res 2003; 63:8302-8311

19. Kouo T, Huang L, Pucsek A, et al. Galectin-3 Shapes Antitumor Immune Responses by Suppressing CD8+ T Cells via LAG-3 and Inhibiting Expansion of Plasmacytoid Dendritic Cells, Cancer Immunol Res 2015; 3(4): 412-423. doi: 10.1158/2326-6066.CIR-14-0150.

20. Fernandez G.C., Ilarregui J.M., Rubel CJ, et al. Galectin-3 and soluble fibrinogen act in concert to modulate neutrophil activation and survival: involvement of alternative MAPK pathways, Glycobiology 2005; 15:519-527. doi:10.1093/glycob/cwi026.

21. Funasaka T, Raz A, Nangia-Makker P. Nuclear transport of galectin-3 and its therapeutic implications. Semin Cancer Biol. 2014; 27:30-8. doi: 10.1016/j.semcancer.2014.03.004.

22. Saravanan C, Liu FT, Gipson IK, Panjwani N. Galectin-3 promotes lamellipodia formation in epithelial cells by interacting with complex $\mathrm{N}$-glycans on alpha3beta1 integrin. $J$ Cell Sci. 2009; 122(20):3684-93. doi: 10.1242/jcs.045674.

23. Zhao Q., Barclay M., Hilkens J, et al. Interaction between circulating galectin-3 and cancer-associated MUC1 enhances tumour cell homotypic aggregation and prevents anoikis, Mol Cancer 2010; 9:154. doi: 10.1186/14764598-9-154.

24. Shekhar MP, Nangia-Makker P, Tait L, Miller F, Raz A. Alterations in galectin-3 expression and distribution correlate with breast cancer progression: functional analysis of galectin-3 in breast epithelial-endothelial interactions. Am J Pathol. 2004; 165(6):1931-41. doi:10.1016/S00029440(10)63245-2

25. Markowska AI, Jefferies KC, Panjwani N. Galectin-3 protein modulates cell surface expression and activation of vascular endothelial growth factor receptor 2 in human endothelial cells. J Biol Chem. 2011; 286(34):2991321. doi: $10.1074 / j b c . M 111.226423$.

26. Wesley UV, Vemuganti R, Ayvaci ER, Dempsey RJ. Galectin-3 enhances angiogenic and migratory potential of microglial cells via modulation of integrin linked kinase signaling. Brain Res. 2013; 1496:1-9. doi: 10.1016/j. brainres.2012.12.008.

27. Yang E, Shim JS, Woo HJ, Kim KW, Kwon HJ. Aminopeptidase N/CD13 induces angiogenesis through interaction with a pro-angiogenic protein, galectin-3. Biochem Biophys Res Commun. 2007; 363(2):336-41. doi:10.1016/j.bbrc.2007.08.179 
28. Jia W, Kidoya H, Yamakawa D, Naito H, Takakura N. Galectin-3 accelerates M2 macrophage infiltration and angiogenesis in tumors. Am J Pathol. 2013; 182(5):182131. doi: 10.1016/j.ajpath.2013.01.017.

29. Eliaz I, McKee D. Galectin-3 as an oncological biomarker. A review of its possible role in cancer treatment response and disease progression. Nat Med J. 2014; 6(9)

30. Liu H, D’Ambrosio M, Liao T, et al. N-acetyl-serylaspartyllysyl-proline prevents cardiac remodeling and dysfunction induced by galectin-3, a mammalian adhesion/growth-regulatory lectin. Am J Physiol Heart Circ Physiol, 2009; 296:H404-412.doi:10.1152/ajpheart.00747.2008.

31. Sharma U, Rhaleb NE, Pokharel S, et al. Novel antiinfl ammatory mechanisms of N-Acetyl-Ser-Asp-LysPro in hypertension-induced target organ damage. Am J Physiol, 2008; 294:H1226-H1232. doi: 10.1152/ajpheart.00305.2007

32. De Boer, RA, van Veldhuisen DJ, Gansevoort RT, et al. The fibrosis marker galectin-3 and outcome in the general population. J Intern Med 2012; 272(1):55-64. doi: 10.1111/j.1365-2796.2011.02476.x

33. Grandin EW, Jarolim P, Murphy SA, et al. Galectin-3 and the development of heart failure after acute coronary syndrome: pilot experience from PROVE IT-TIMI 22. Clin Chem, 2012; 58(1):267-273. doi: 10.1373/ clinchem.2011.174359

34. Lok DJ, Van Der Meer P, de la Porte PW, et al. Prognostic value of galectin -3 , a novel marker of fi brosis, in patients with chronic heart failure: data from the DEAL - HF Study. Clin Res Cardiol. 2010; 99:323-328. doi: 10.1007/s00392-010-0125-y.

35. De Boer RA, Lok DJ, Jaarsma T, et al. Predictive value of plasma galectin -3 levels in heart failure with reduced and preserved ejection fraction. Ann Med, 2011; 43:60-68. doi:10.3109/07853890.2010.538080.

36. De Filippi C., Christenson R., Shah R, et al. Clinical validation of a novel assay for galectin-3 for risk assessment in acutely destabilized heart failure. J Card Fail, 2009; 15:S9. doi: 10.1016/j.cardfail.2009.06.405

37. Ho JE, Yin X, Levy D, et al. Galectin 3 and incident atrial fibrillation in the community. Am Heart $J$ 2014: 167:729-734 e721. doi: 10.1016/j.ahj.2014.02.009.

38. Sonmez O, Ertem FU, Vatankulu MA, et al. Novel fibroinflammation markers in assessing left atrial remodeling in nonvalvular atrial fibrillation. Med Sci Monit 2014; 20:463-470. doi:10.12659/MSM.890635.
39. Wu XY, Li SN, Wen SN, et al. Plasma galectin-3 predicts clinical outcomes after catheter ablation in persistent atrial fibrillation patients without structural heart disease. Europace. 2015; 17:1541-1547. doi: 10.1093/europace/ euv045.

40. Kornej J., Schmidl J., Ueberham L, et al. Galectin-3 in Patients with Atrial Fibrillation Undergoing Radiofrequency Catheter Ablation. PLoS ONE. 2015; 10(4):e0123574. doi:10.1371/journal.pone.0123574.

41. He B., Huang B, Lu Z, He W, Jiang H. Galectin-3: a potential new target for upstream therapy of atrial fibrillation. Int J Cardiol 2016; 203:1131-1132. doi:10.1016/j. ijcard.2015.09.058.

42. Chen D, Procter N, Goh V, Liu S, Chua SJ, AssadiKhansari B, et al. New onset atrial fibrillation is associated with elevated galectin-3 levels. Int J Cardiol. 2016: 223, 48-49. doi: 10.1016/j.ijcard.2016.08.172

43. Clementy N, Piver E, Benhenda N, Bernard A, Pierre B, Simeon E, et al. Galectin-3 in patients undergoing ablation of atrial fibrillation . IJC Metab Endocr 2014: http:// dx.doi.org/10.1016/j.ijcme.2014.10.003.

44. Kisheva A. Role of fibrosis and blocking of mineralcorticoid receptors in atrial fibrillation. PhD Thesis, 2017, Medical University, Varna, Bulgaria.

45. Bouguenina H, Salaun D, Mangon A, Muller L, Baudelet E, Camoin L, et al. EB 1-binding -myomegalin protein complex promotes centrosomal microtubules functions . Proc Natl Acad Sci USA . 2017 ;114(50):E 10687-E10696. doi: 10.1073/pnas.1705682114.

46. Clare DK, Magescas J, Piolot T, Dumoux M, Vesque C, Pichard E, et al. Basal foot MTOC organizes pillar MTs required for coordination of beating cilia. Nat Commun 2014;5:4888. doi: 10.1038/ncomms5888.

47. Albrethsen J, Angeletti RH, Horwitz SB, Yang CP. Proteomics of cancer cell lines resistant to microtubulestabilizing agents. Mol Cancer Ther 2014;13(1):260-269. doi: 10.1158/1535-7163.MCT-13-0471

48. Yanev S, Fiore M, Hinev A, Ghenev PI, Hristova MG, Panayotov $\mathrm{P}$, et al. From antitubulins to trackins. Biomed Rev 2016;27: 59-67.

49. Natale A, Elayi CS. Colchicine for prevention of post cardiac procedure atrial fibrillation: Meta - analysis of randomized controlled trials. Int J Cardiol 2017; 243:258 -262 .

50. Chaldakov GN. Colchicine, a microtubule-disassembling drug, in the therapy of cardiovascular diseases. Cell Biol Int 2018;42(8):1079-1084. doi:10.1002/cbin.10988 